\title{
The Quantitative Density Measurement of Unsteady Flow around the Projectile*
}

\author{
Yusuke Hirose", Kazuki Ishikawa1, Yoshiyuki Ishimoto', Takumi Nagashima1, Masanori Ota', \\ Shinsuke Udagawa², Tatsuro Inage ${ }^{3}$, Kazuki Fujita3 ${ }^{3}$, Hideki Kiritani', Koji Fujita4, \\ Kiyonobu Ohtani ${ }^{4}$, Hiroki Nagai ${ }^{4}$
}

${ }^{1}$ Graduate School of Engineering, Chiba University, Chiba, Japan

${ }^{2}$ Aerospace Engineering Course, Tokyo Metropolitan College of Industrial Technology, Tokyo, Japan

${ }^{3}$ Department of Mechanical Engineering, Shonan Institute of Technology, Fujisawa, Kanagawa, Japan

${ }^{4}$ Institute of Fluid Science, Tohoku University, Sendai, Miyagi, Japan

Email: ota@faculty.chiba-u.jp

How to cite this paper: Hirose, Y., Ishikawa, K., Ishimoto, Y., Nagashima, T., Ota, M., Udagawa, S.., Inage, T., Fujita, K., Kiritani, H., Fujita, K., Ohtani, K. and Nagai, H. (2019) The Quantitative Density Measurement of Unsteady Flow around the Projectile. Journal of Flow Control, Measurement \& Visualization, 7, 111-119. https://doi.org/10.4236/jfcmv.2019.72009

Received: December 17, 2018

Accepted: February 20, 2019

Published: April 28, 2019

Copyright $\odot 2019$ by author(s) and Scientific Research Publishing Inc. This work is licensed under the Creative Commons Attribution International License (CC BY 4.0).

http://creativecommons.org/licenses/by/4.0/

\begin{abstract}
Three-dimensional density measurement of unsteady flow field around a sphere is carried out in the ballistic range at Institute of Fluid Science, Tohoku University. Simultaneous multi-angle measurement system using twelve digital cameras is installed in the test chamber of the ballistic range to achieve the three-dimensional density measurement. The Colored-Grid Background Oriented Schlieren (CGBOS) technique using colored-grid background is utilized for the reconstruction of density. The Mach number of the sphere is set to 1.5. The short pulse LEDs to illuminate the backgrounds are also installed in the chamber to capture the unsteady flow field around a flying sphere. Three-dimensional density distribution around a sphere is successfully reconstructed.
\end{abstract}

\section{Keywords}

Background Oriented Schlieren, Flow Visualization, High Speed Flows, Unsteady Flow, Simultaneous Measurement

\section{Introduction}

The supersonic aircrafts and rockets are developed actively all over the world. In the development process, the density measurement around the model of aircraft and rockets under the supersonic speed is important in order to obtain aerodynamic properties [1] [2] [3].

${ }^{*} \mathrm{~A}$ part of this work was presented at 15th International Conference Fluid Dynamics (ICFD15) in Sendai, 7-9 Nov. 2018. 
Conventionally, the density measurement is achieved by using Schlieren or shadowgraph technique. The principle of Schlieren technique is well known; it exploits the bending of light ray caused by a change of the refractive index corresponding to the density change in the medium. The technique is sensitive to the density gradient. However, the technique is mainly applied to qualitative density measurement. Furthermore, the technique employs many optical elements, such as a pinhole, concave mirror, knife-edge, color filter, camera, etc. and needs precise adjustment [4].

Recently, the Background Oriented Schlieren (BOS) technique is proposed by Meier [5]. The BOS technique enables us to take a quantitative density measurement of flow field with computer-aided image analysis. In contrast to the conventional Schlieren technique, the BOS technique requires only a background and a digital camera to realize the quantitative measurement of density. In the past several years, the BOS technique has been applied in various experiments [6] [7]. Also, Ota et al. proposed the Colored-Grid Background Oriented Schlieren (CGBOS) technique that uses a colored-grid background and the technique has been applied to some experiments [8]. Ota et al. also aimed the three-dimensional density measurements by using CGBOS technique. The previous reports have focused on the reconstruction of steady flow, reproducible unsteady flow or axially-symmetrical unsteady flow in the wind tunnel test because the flow field was measured with one camera from one direction and multi-projections of flow were obtained with repetition of many blow-downs [9] [10] or one projection under axially-symmetric condition [11]. However, the unsteady phenomena should be measured simultaneously from multi-projection angles because the "real" phenomena would never happen again.

In this study, twelve digital cameras are employed to realize the simultaneous multi-angle observation and reconstruction of the unsteady flow around the projectile in the ballistic range experiments. The digital cameras and LEDs are used to construct the multiangle CGBOS measurement system with low-cost. Unlike common wind tunnels, the supports of the experimental model are not necessary for free-flight test in the ballistic range; therefore the phenomena in the wake flow region can also be measured. The Schlieren visualization or the measurements with pressure sensors have been mainly carried out in the ballistic range experiments so far and three-dimensional density measurements are rarely performed. In this paper, three-dimensional unsteady flow field around a sphere (Diameter $\phi=15 \mathrm{~mm}$ ) including its wake region is measured by the CGBOS technique at the ballistic range in Institute of Fluid Science (IFS), Tohoku University.

\section{Experimental Setup, Condition and Method}

Figure 1 shows an optical setup for the BOS technique. The setup is composed of a background image, a lens and an image sensor where $l_{b}$ denotes the distance from the background image to the density gradient, $l_{c}$ the distance from density 


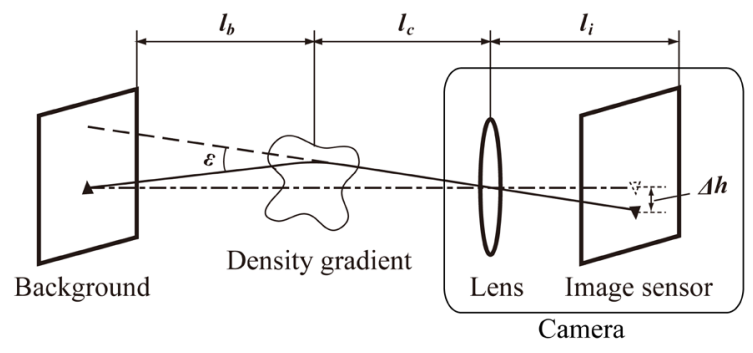

Figure 1. Optical setup for BOS measurement.

gradient to lens, $l_{i}$ the distance from lens to image sensor, $\varepsilon$ the refraction angle and $\Delta h$ the displacement of the background. When the density in the medium is changed between the background image and the image sensor, the background image is captured at the image sensor with displacement $\Delta h$ because of the refraction of light passing through the density gradients, shown as a solid line in the figure. The relation between the displacement of the background $\Delta h$ and refractive index $n$ is expressed as Equation (1), where $f$ is the focal length of the camera, and $n_{0}$ is reference refractive index [5]. The relation between density $\rho$ and refractive index $n$ is given by the Gladstone-Dale equation expressed as Equation (2), where $G$ is the Gladstone-Dale constant.

The schematic diagram of the ballistic range in IFS is shown in Figure 2. This apparatus can eject the projectile at $300 \mathrm{~m} / \mathrm{s}$ to $700 \mathrm{~m} / \mathrm{s}$ in gas gun mode. In this paper, twelve digital cameras (Canon EOS Kiss Digital X 3, $4752 \times 3168$ pixels) with telephoto zoom lenses (SIGMA 70 - $300 \mathrm{~mm}$ F4-5.6 DG MACRO), the backgrounds and LEDs were set in the test section placed with $15^{\circ}$ intervals as shown in Figure 3 and Figure 4. Since the ballistic range shown in Figure 2 has the test-section chamber which has the inner diameter of $1660 \mathrm{~mm}$, it is necessary to set the camera at the position as close to the inner wall of the test section as possible while securing the space for setting and adjusting the position of the camera. The position of the background was determined from the shortest focal distance of the used camera lens, therefore, the distance from a camera to a background $\left(l_{b}+I_{c}\right)$ is set to $950 \mathrm{~mm}$ and the distance from the background to the trajectory of a sphere $I_{b}$ is set to $255 \mathrm{~mm}$ as shown in Figure 3. The focal length of zoom lens is set to $190 \mathrm{~mm}$ considering the size of the field of vision. The duration of the LEDs flushes is set to $5 \mathrm{~ns}$ and it is desirable that the value of $F$-number of camera lends should be higher although the luminescence time was too short to obtain the adequate brightness. The spatial resolution for the observed phenomena will be lower in the case of decreasing $F$-number, i.e. opening the aperture of the lens, because the cone diameter of the incident light from the background to the camera lens at the observation position becomes larger [12]. Thus, $F$-number was set to be close to the maximum value $(F=18)$ by increasing ISO sensitivity of the camera.

The colored-grid background is composed of blue and red stripes. The red stripes are used for the horizontal direction and the blue for the vertical as shown in Figure 4. The background images are illuminated by white LEDs from 


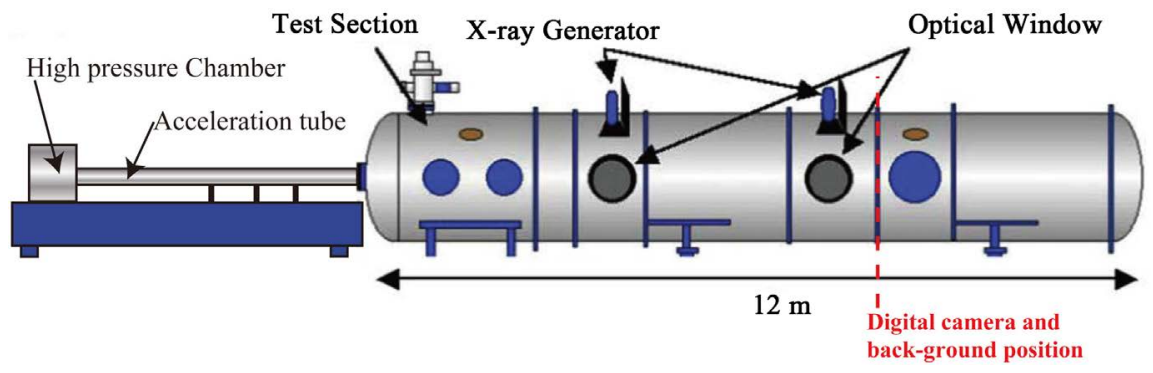

Figure 2. The ballistic range in IFS, Tohoku University (gas gun mode, from IFS Home page).

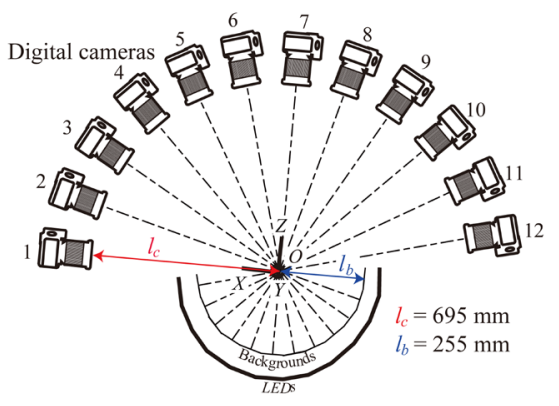

Figure 3. Schematic of simultaneous measurement system.
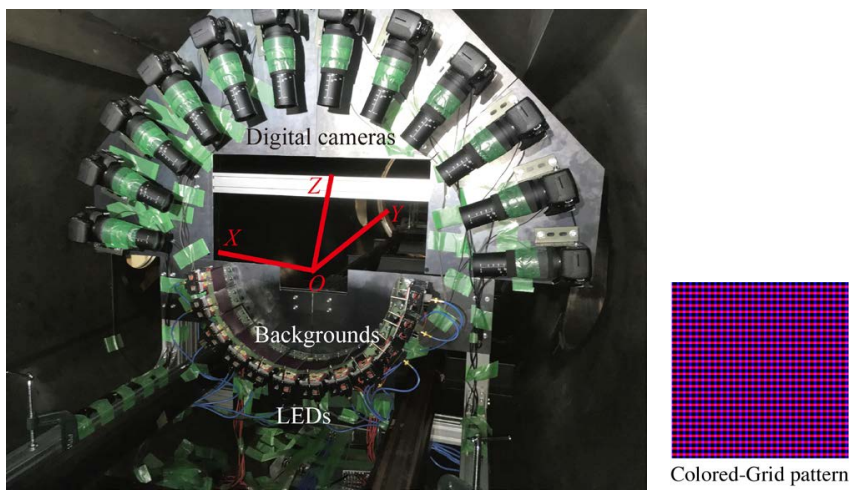

Figure 4. Twelve cameras, backgrounds and LEDs installed in the test chamber.

the back of them. Four $10 \mathrm{~W}$-LEDs are used for one background image, therefore totally $480 \mathrm{~W}$ LEDs are installed. For the comparison, the conventional Schlieren measurement by high speed camera (Shimazu HPV-X) was also carried out. The projectile is a sphere with a diameter of $15 \mathrm{~mm}$ and its flight speed is Mach 1.5. The temperature and pressure of atmosphere is $300 \mathrm{~K}$ and 101.3 $\mathrm{kPa}$. The Reynolds number based on the diameter of a sphere is $4.98 \times 10^{5}$. The frame rate and exposure time of the high-speed camera are set to 100,000 fps (frames per second) and $1.0 \mu \mathrm{s}$. In this paper, the ART (Algebraic Reconstruction Technique) [13] is employed for the reconstruction of density gradients. The basic equation of ART is shown in Equation (3), where $i$ is the number of the iteration, $f^{i}(x, y)$ is density gradient in this paper, $P(r, \theta)$ is projection data obtained from experiment, $C(r, \theta)$ is number of pixels in a projection path and $R^{i-1}(r, \theta)$ is pseudo-projection data calculated from $f^{i-1}(x, y)$ [13]. Then the den- 
sity distribution is obtained from the reconstructed density gradient by solving Successive Over Relaxation (SOR) method. The methodologies of the reconstruction are same as our previous report [8]. The relation between projection angle $\theta$ and image plane is shown in Figure 5. As mentioned above the unsteady flow field around a flying sphere is reconstructed from 12 view angles with $15^{\circ}$ intervals. In this study the experiment using 12 cameras is the most efficient condition. Because the smaller number of the view angle, the less accuracy of the reconstruction results. The reconstruction would be more accurate with larger number of view angles, however the space in the test chamber is limited. The Gladstone-Dale constant in the reconstruction is set to $2.26 \times 10^{-4} \mathrm{~m}^{3} / \mathrm{kg}$ for an average wavelength $\lambda$ in the visible spectral range $(\lambda=550 \mathrm{~nm})$.

$$
\begin{gathered}
\Delta h=\frac{l_{b} f}{l_{b}-l_{c}-f} \frac{1}{n_{0}} \int_{l_{b}-\Delta l_{b}}^{l_{b}+\Delta l_{b}} \frac{\partial n}{\partial r(x, y)} \mathrm{d} l \\
n=\rho G+1 \\
f^{i}(x, y)=f^{i-1}(x, y)+\frac{P(r, \theta)-R^{i-1}(r, \theta)}{C(r, \theta)}
\end{gathered}
$$

\section{Experimental Results}

Figure 6 shows the conventional Schlieren image captured by the high-speed camera with horizontal knife-edge. From this image, Bow shock, expansion wave, separation shock and reattachment shock can be seen. A red rectangle in Figure 6 indicates capturable size for the CGBOS measurement $(60 \mathrm{~mm} \times 40$ $\mathrm{mm}$ ). A CGBOS image taken from a digital camera (No. 1 in Figure 3) is shown in Figure 7 where a red rectangle indicates the processed region for the reconstruction. In this paper $457 \times 689 \times 457$ voxels are used, which corresponds to a distance of $0.07 \mathrm{~mm}$ between the voxel centroids. The CGBOS images are captured by emitting the LEDs when a sphere passed through the photographable region while the shutters of the digital cameras are being opened. However, it is

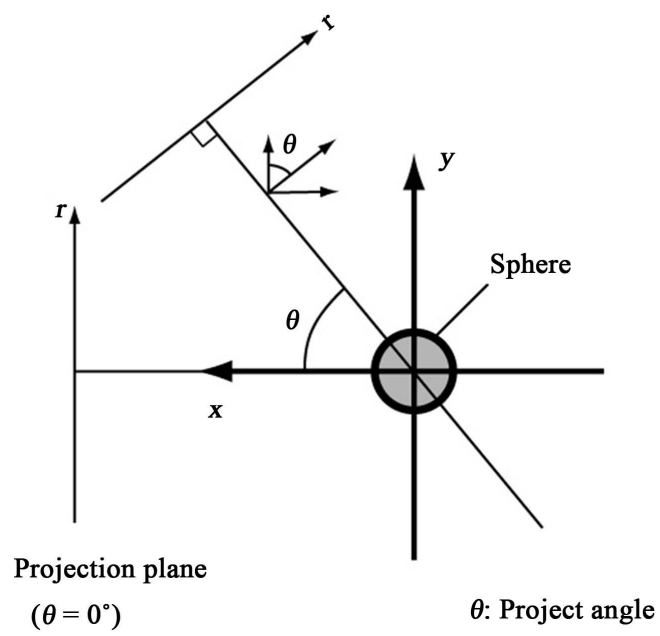

Figure 5. Projection angle for reconstruction. 


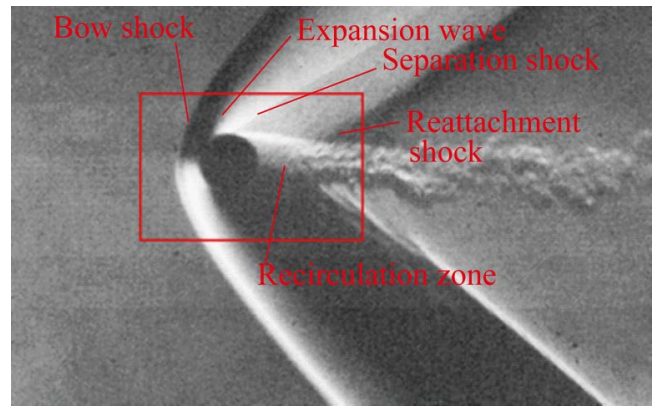

Figure 6. Schlieren image.

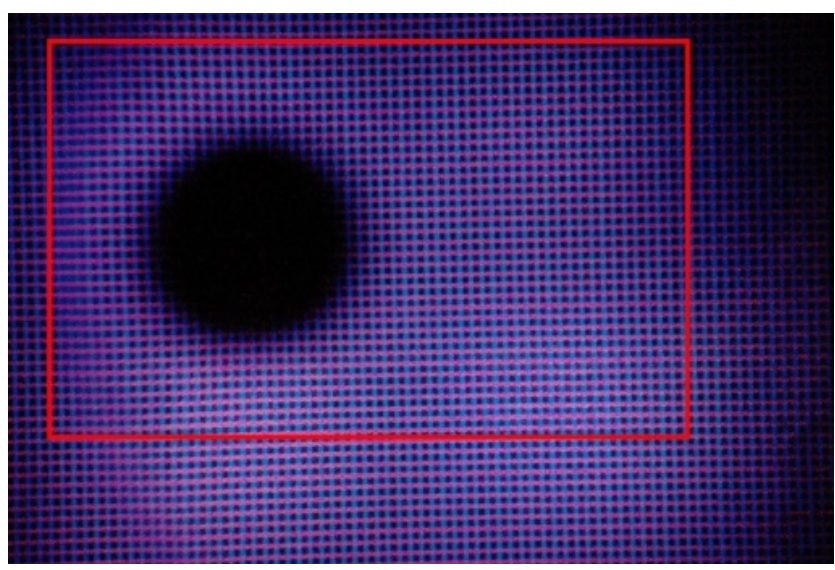

Figure 7. An example of CGBOS image.

necessary that the timing of emitting the LEDs should be strictly adjusted because the photographable region is small as shown in Figure 6. Figure 8 shows the vertical displacements obtained from CGBOS images of camera 1 and 7. The captured phenomena are almost same because the most of flow around sphere is axisymmetric except the wake region behind a sphere. The bow shock in front of sphere and expansion wave are captured, however the separation shock cannot be seen clearly this is because the observed phenomena contain blurs due to the diverging light observation with normal camera lens [10].

Three-dimensional density distribution around a sphere is reconstructed from 12 CGBOS images captured simultaneously. As mentioned above, after the reconstruction of the density gradient by ART, three-dimensional density distribution is obtained by solving the Poisson equation using the successive over relaxation method [8]. Reconstructed density distribution on the central plane is shown in Figure 9. Bow shock in front of a sphere, recirculation zone and reattachment shock in the wake region are captured. The low-density region around recirculation zone seems to be thicker than that of the conventional schlieren image in Figure 6 this is because the error of ART reconstruction and integration process using SOR method, furthermore captured CGBOS images are blurred due to the diverging light observation with BOS measurement [10]. Figure 10 shows isopycnic surface of free-stream density ratios 0.89 and 1.08. The flow in the wake of the sphere can be clearly seen. Artifacts (noises) around a 


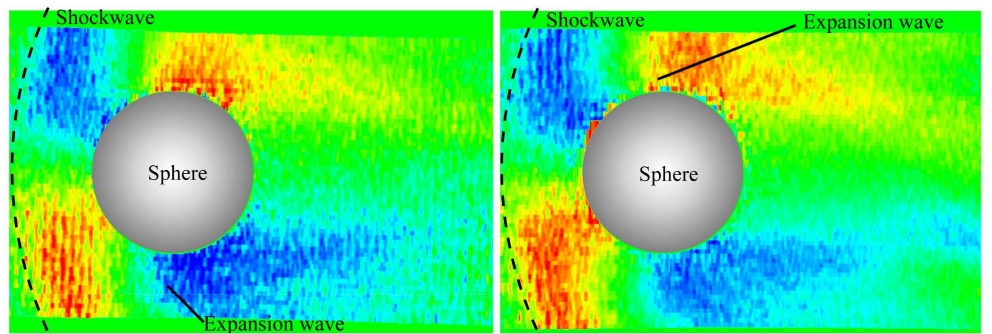

Figure 8. Vertical displacement (left: camera 1, right: camera 7).

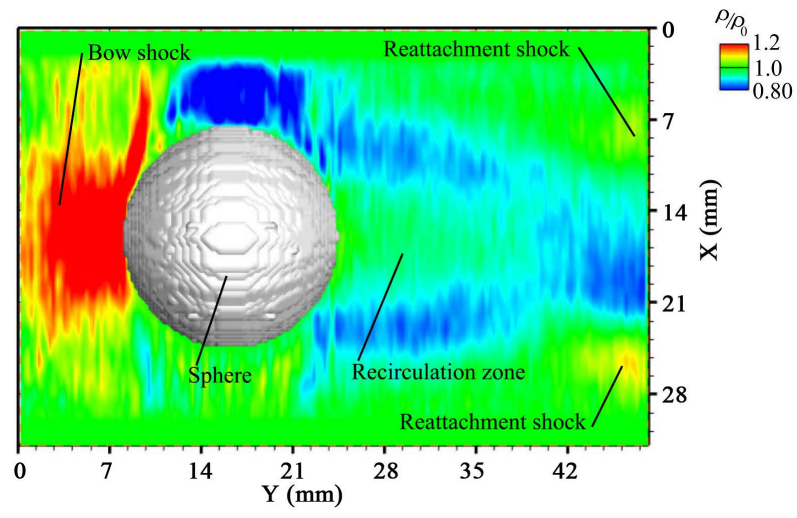

Figure 9. Reconstructed density distribution on central plane.

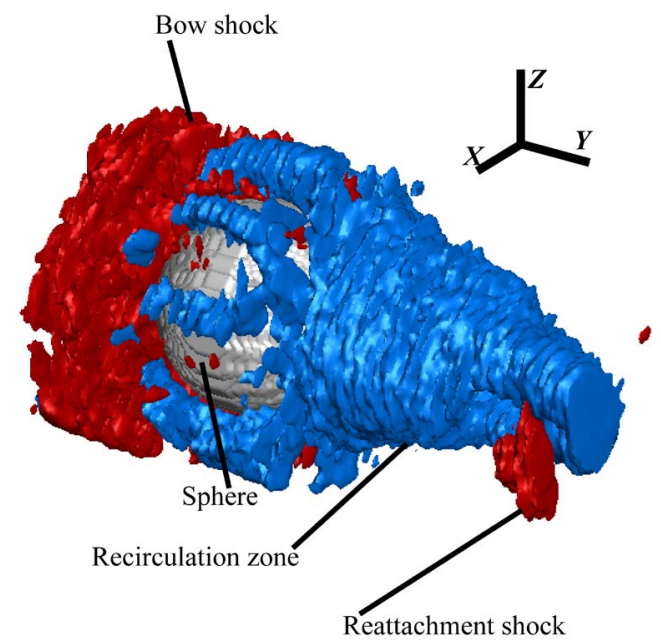

Figure 10. Isopycnic surface (Red: $\rho / \rho_{0}=1.08$, Blue: $\rho / \rho_{0}=0.89$ ).

sphere can be confirmed, this is because the background image cannot be captured in the sphere position and the reconstruction has to be done from insufficient projection data. However, there is no obstacle in the wake region, the reconstructed results, e.g. the $3 \mathrm{D}$ shape of recirculation zone and reattachment shock wave can be reliable.

\section{Conclusion}

The simultaneous multi-angle measurement system using the BOS technique is installed in the ballistic range at Institute of Fluid Science, Tohoku University. In 
order to capture the unsteady flow field around a flying sphere at supersonic speed, twelve digital cameras and short pulse LEDs are introduced in the system. We succeeded in capturing CGBOS images simultaneously from 12 directions and the reconstruction of three-dimensional density distribution. As a result, unsteady flow field around a flying sphere at the speed of Mach 1.5 is successfully reconstructed. Especially, the wake flow of the flying sphere is captured clearly such as the recirculation zone and the reattachment shock. The quantitative density measurement of the unsteady wake flow becomes possible by the proposed measurement system.

\section{Acknowledgements}

Part of the work was carried out under the Collaborative Research Project of the Institute of Fluid Science, Tohoku University.

\section{Conflicts of Interest}

The authors declare no conflicts of interest regarding the publication of this paper.

\section{References}

[1] Rausch, V.L., McClinton, C.R. and Crawford, J.L. (1997) Hyper-X: Flight Validation of Hypersonic Airbreathing Technology. ISABE, 97-7024.

[2] Joslin, R.D. (1998) Aircraft Laminar Flow Control. Annual Review of Fluid Mechanics, 30, 1-29.

[3] Carlson, H.W. (1964) Correlation of Sonic-Boom Theory with Wind-Tunnel and Flight Measurements. NASA Technical Report NASA TR R-213.

[4] Settles, G.S. (1995) Full-Scale Schlieren Flow Visualization. The 7 th International Symposium on Flow Visualization, Seattle, 11-14 September 1995, 2-13.

[5] Meier, G.E.A. (2002) Computerized Background-Oriented Schlieren. Experiments in Fluids, 33, 181-187. https://doi.org/10.1007/s00348-002-0450-7

[6] Leopold, F., Simon, J., Gruppi, D. and Schäfer, H.J. (2006) Recent Improvements of the Background Oriented Schlieren Technique (BOS) by Using a Colored Background. International Symposium on Flow Visualization, Göttingen, ISFV12-3.4, $1-10$.

[7] Venkatakrishnan, L. and Meier, G.E.A. (2004) Density Measurements Using the Background Oriented Schlieren Technique. Experiments in Fluids, 37, 237-247. https://doi.org/10.1007/s00348-004-0807-1

[8] Ota, M., Hamada, K., Kato, H. and Maeno, K. (2011) Computed-Tomographic Density Measurement of Supersonic Flow Field by Colored-Grid Background Oriented Schlieren (CGBOS) Technique. Measurement Science and Technology, 22, 1-7. https://doi.org/10.1088/0957-0233/22/10/104011

[9] Ota, M., Kurihara, K., Aki, K., Miwa, Y., Inage, T. and Maeno, K. (2015) Quantitative Density Measurement of the Lateral Jet/Cross Flow Interaction Field by Colored-Grid Background Oriented Schlieren (CGBOS) Technique. Journal of Visualization, 18, 543-552. https://doi.org/10.1007/s12650-015-0297-7

[10] Ota, M., Leopold, F., Noda, R. and Maeno, K. (2015) Improvement in Spatial Resolution of Background-Oriented Schlieren Technique by Introducing a Telecentric 
Optical System and Its Application to Supersonic Flow. Experiments in Fluids, 56, 1-10. https://doi.org/10.1007/s00348-015-1919-5

[11] Leopold, F., Ota, M., Klatt, D. and Maeno, K. (2013) Reconstruction of the Unsteady Supersonic Flow around a Spike Using the Colored Background Oriented Schlieren Technique. Journal of Flow Control, Measurement \& Visualization, 1, 69-76. https://doi.org/10.4236/jfcmv.2013.12009

[12] Ardian, B.G., Burim, K. and Shigeru, O. (2013) Measurement Sensitivity and Resolution for Background Oriented Schlieren During Image Recording. Journal of Visualization, 16, 201-207. https://doi.org/10.1007/s12650-013-0170-5

[13] Kak, A.C. and Slaney, M. (1988) Principle of Computerized Tomographic Imaging. IEEE Press, New York.

\section{Nomenclature}

$C(r, \theta)$ : Number of pixels in projection path

f. Focal length of camera [mm]

$f(x, y)$ : Density gradient

$G$ : Gladstone-Dale constant $\left[\mathrm{m}^{3} / \mathrm{kg}\right]$

$l_{b}$ : Distance from the background image to the density gradient $[\mathrm{m}]$

$l_{c}$ : Distance from density gradient to lens $[\mathrm{m}]$

$l_{i}$ : Distance from lens to image sensor $[\mathrm{m}]$

$n$ : Refractive index [-]

$n_{0}$ : Reference refractive index [-]

$P(r, \theta)$ : Projection data obtained from experiment

$R^{i-1}(r, \theta)$ : Pseudo-projection data calculated from $f^{-1}(x, y)$

$\varepsilon$ : Refraction angle [deg]

$\theta$. Projection angle $[\mathrm{deg}]$

$\lambda$ : Average wavelength $[\mathrm{nm}]$

$\rho$ : Density $\left[\mathrm{kg} / \mathrm{m}^{3}\right]$

$\Delta h$ : Displacement of background [m]

$\rho / \rho_{0}$ : Density ratio [-] 